> Le suppresseur de tumeurs p53 est un médiateur essentiel des voies de réponse cellulaire à des stress de nature très variée qui est inactivé dans la majorité des tumeurs. Au-delà de ses fonctions connues dans le contrôle de la prolifération, de la sénescence ou de la mort cellulaire, des résultats récents de différents laboratoires indiquent que p53 joue de multiples rôles dans le contrôle du métabolisme énergétique, et qu'il est lui même régulé par de nombreux changements métaboliques. II est vraisemblable que ces actions de p53, encore mal connues, jouent un rôle important, à la fois pour les fonctions de suppresseur de tumeurs de cette protéine, mais également dans l'homéostasie tissulaire, ainsi qu'au cours du vieillissement. <

p53 est l'archétype du gène suppresseur de tumeurs dont l'inactivation promeut le développement de nombreux types tumoraux. Des mutations de TRP53 (transformation related protein 53 ), le gène codant pour la protéine p53, ont été identifiées dans environ $50 \%$ des tumeurs humaines, alors que l'autre moitié comporte des altérations génétiques de régulateurs situés en amont ou aval de p53 conduisant, in fine, à l'inactivation fonctionnelle de la cascade moléculaire dans laquelle intervient $p 53$. p53 est un facteur de transcription qui régule l'expression de plusieurs centaines de gènes ou ARN non codants cibles. p53 est considéré comme un médiateur essentiel des mécanismes de réponse cellulaire à des stress de nature très variée, tels que les stress génotoxiques, le stress oxydant, l'hypoxie ou encore le stress du réticulum endoplasmique. L'activation de p53, dans le contexte de ces stress aigus, conditionne le destin cellulaire et peut conduire, soit à un arrêt transitoire de la prolifération cellulaire et de la réparation des dommages, soit, lorsque ces dommages sont trop importants, à un arrêt irréversible de la prolifération cellulaire appelé sénescence [38] ou encore à la mort cellulaire. II apparaît cependant que si p53 joue un rôle essentiel dans ces situations de stress

Vignette (Photo @ Inserm - Jérome Galon).

\section{Rôle \\ du suppresseur \\ de tumeurs p53 \\ dans le contrôle \\ du métabolisme}

Matthieu Lacroix, Laetitia Karine Linares, Laurent Le Cam

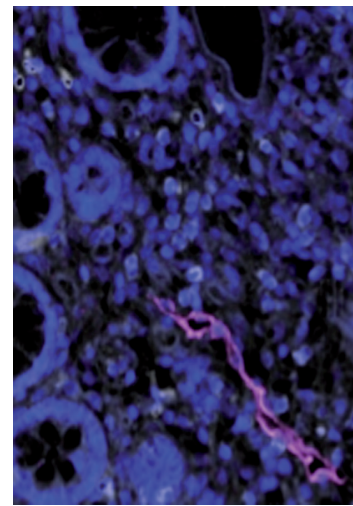

Institut de recherche en cancérologie de Montpellier, Inserm U896, Institut régional de lutte contre le cancer (ICM), université Montpellier 1, 208, rue des apothicaires, 34298 Montpellier Cedex, France. laurent.lecam@inserm.fr matthieu.lacroix@inserm.fr laetitia.linares@inserm.fr

aigu, cette protéine n'en est pas moins impliquée dans des réponses adaptatrices plus subtiles permettant à la cellule de s'adapter à son environnement, notamment en termes de disponibilité en nutriments. À l'instar d'autres réseaux moléculaires dans lesquels p 53 intervient, les effets de p53 sur le métabolisme sont multiples et dépendent, non seulement du type cellulaire, mais également de la nature et de l'intensité des perturbations qui ont conduit à son activation (dommages à l'ADN, stress oxydant, perturbations métaboliques, etc.). Les rôles de $p 53$ dans le contrôle du métabolisme impliquent la régulation de certains de ses gènes cibles, mais passent également par des effets non transcriptionnels encore mal élucidés.

\section{p53 intervient dans de nombreuses voies métaboliques}

p53 est capable d'activer - mais également de réprimer - la transcription de nombreux gènes clés du métabolisme, modulant in fine la glycolyse, la voie des pentoses phosphates, la phosphorylation oxydative, le métabolisme des lipides ou celui de certains acides aminés non essentiels, tels que la glutamine ou la sérine (Tableau I, Figure 1). p53 intervient également dans certaines cascades de signalisation qui jouent de multiples rôles dans le métabolisme, comme la voie IGF (insulin-like growth factor)-PI3K (phosphoinositide 3-kinase)-mTOR (mammalian target of rapamycin) (Tableau I).

p53 et la voie IGF-PI3K-mTOR

Certains gènes cibles de $p 53$ constituent des éléments importants de la voie IGF-PI3K-mTOR, une des voies de signalisation majeure 


\begin{tabular}{|c|c|c|}
\hline \multirow{5}{*}{ Statut redox } & Gènes réprimés & Gènes activés \\
\hline & Cox2 [27] & Gpxl [27] \\
\hline & Nos2 [27] & Sod2 [27] \\
\hline & & Aldh4 [27] \\
\hline & & Sesl, $2[3,4]$ \\
\hline \multirow{12}{*}{$\begin{array}{l}\text { Métabolisme } \\
\text { des lipides }\end{array}$} & Srebpl [17] & Lpin [15] \\
\hline & $\mathrm{Mel}, 2$ [29] & Gamt [14] \\
\hline & & Crot $[16]$ \\
\hline & & Cptla [16] \\
\hline & & Cptlc [16] \\
\hline & & $\mathrm{Npcl} / \mathrm{l}[\mathrm{l} 6]$ \\
\hline & & Pltp [16] \\
\hline & & $\mathrm{Cel}[16]$ \\
\hline & & Abcal2 [16] \\
\hline & & Acer2 [16] \\
\hline & & Apobecl [16] \\
\hline & & Dhrs3 [16] \\
\hline Glutaminolyse & & Gls2 $[19,20]$ \\
\hline Glycolyse & Glut1, 4 [7] & Tigar [9] \\
\hline & & $H k 2[13]$ \\
\hline $\begin{array}{l}\text { OxPhos et biogenèse } \\
\text { des mitochondries }\end{array}$ & $\operatorname{Pgcl} \alpha[35]$ & $S \operatorname{co2}[10]$ \\
\hline
\end{tabular}

Tableau I. Gènes du métabolisme réprimés ou induits par p 53.

du contrôle du métabolisme. Les liens entre p53 et cette voie de signalisation sont multiples, impliquant notamment la régulation transcriptionnelle des gènes codant pour les sous-unités $\beta 1$ et $\beta 2$ de l'AMPK (une kinase activée par la carence en nutriments, tels qu'une carence en glucose ou en certains acides aminés, ou par une augmentation du ratio AMP/ATP) [1], TSC2 (tuberous sclerosis 2) (un régulateur négatif de la kinase mTOR) [1], PTEN (la phosphatase qui déphosphoryle le phosphatidylinositol 3-phosphate [PIP3] en PIP2, et s'oppose ainsi à l'activation de la PI3K) [2] ou encore I'IGFBP3 (une molécule sécrétée capable de lier les facteurs de croissance de la famille de l'IGF) [1]. Par ailleurs, les gènes codant pour les sestrines 1 et 2 sont activés par p53, notamment dans des situations de stress oxydant [3, 4]. Au-delà de leur rôle comme molécules anti-oxydantes, les sestrines sont également des inhibiteurs du complexe impliquant la kinase mTORCl [5]. Enfin, la phosphorylation de l'oncogène MDM2 (mouse double minute 2), un des régulateurs négatifs essentiels de p53, par la kinase AKT (protein kinase $B$ ), illustre la notion selon laquelle les liens entre la voie IGF-PI3K-mTOR et la voie p53 sont multiples et s'étendent au-delà de la régulation de $\mathrm{p} 53$ lui-même [6].

\section{Régulation de la glycolyse et de la respiration mitochondriale}

Si les effets de p53 sur le métabolisme restent encore mal compris, plusieurs observations convergent pour suggérer que $p 53$ inhibe la glycolyse via différents mécanismes. Les effets de p53 sur la glycolyse impliquent la répression transcriptionnelle directe ou indirecte des transporteurs du glucose Glutl, Glut3 et Glut4 [7, 8], mais aussi l'activation transcriptionnelle de TIGAR (tumor protein 53-induced glycolysis and apoptosis regulator), une fructose 2,6-bisphophatase qui favorise le flux d'intermédiaires de la glycolyse vers la branche oxydative de la voie des pentoses phosphates [9].

De façon vraisemblablement coordonnée, p53 favorise la respiration mitochondriale à travers l'activation de SCO2 (synthesis of cytochrome c oxydase) [10], ainsi que la biosynthèse et le bon fonctionnement des mitochondries en régulant directement TFAM (transcription factor A, mitochondrial) [11] et la polymérase gamma [12]. À ce titre, certains auteurs ont suggéré que l'inactivation fonctionnelle de p53 au cours de la transformation cellulaire pourrait participer à l'effet Warburg, une transition métabolique bien caractérisée qui favorise la glycolyse au détriment de la phosphorylation oxydative mitochondriale. Cependant, certaines données de la littérature suggèrent un effet paradoxal de p53 sur la glycolyse, notamment à travers l'activation transcriptionnelle de l'hexokinase 2 (HK2), qui code pour une enzyme qui catalyse la première étape de la glycolyse [13]. Ces effets, en apparence contradictoires, pourraient se justifier par la promotion d'un flux d'intermédiaires de la glycolyse vers d'autres voies anaboliques, telles que la branche oxydative de la voie des pentoses phosphates, qui favorise la génération d'équivalents réducteurs (NADPH) qui participent aux défenses cellulaires anti-oxydantes en réduisant le glutathion, et également à la biosynthèse des lipides et de certains précurseurs des acides nucléiques.

\section{Régulation du métabolisme des lipides}

p53 est au cœur de boucles de rétrocontrôle impliquées dans le contrôle du métabolisme des lipides. De fait, p53 favorise l'utilisation des lipides comme 


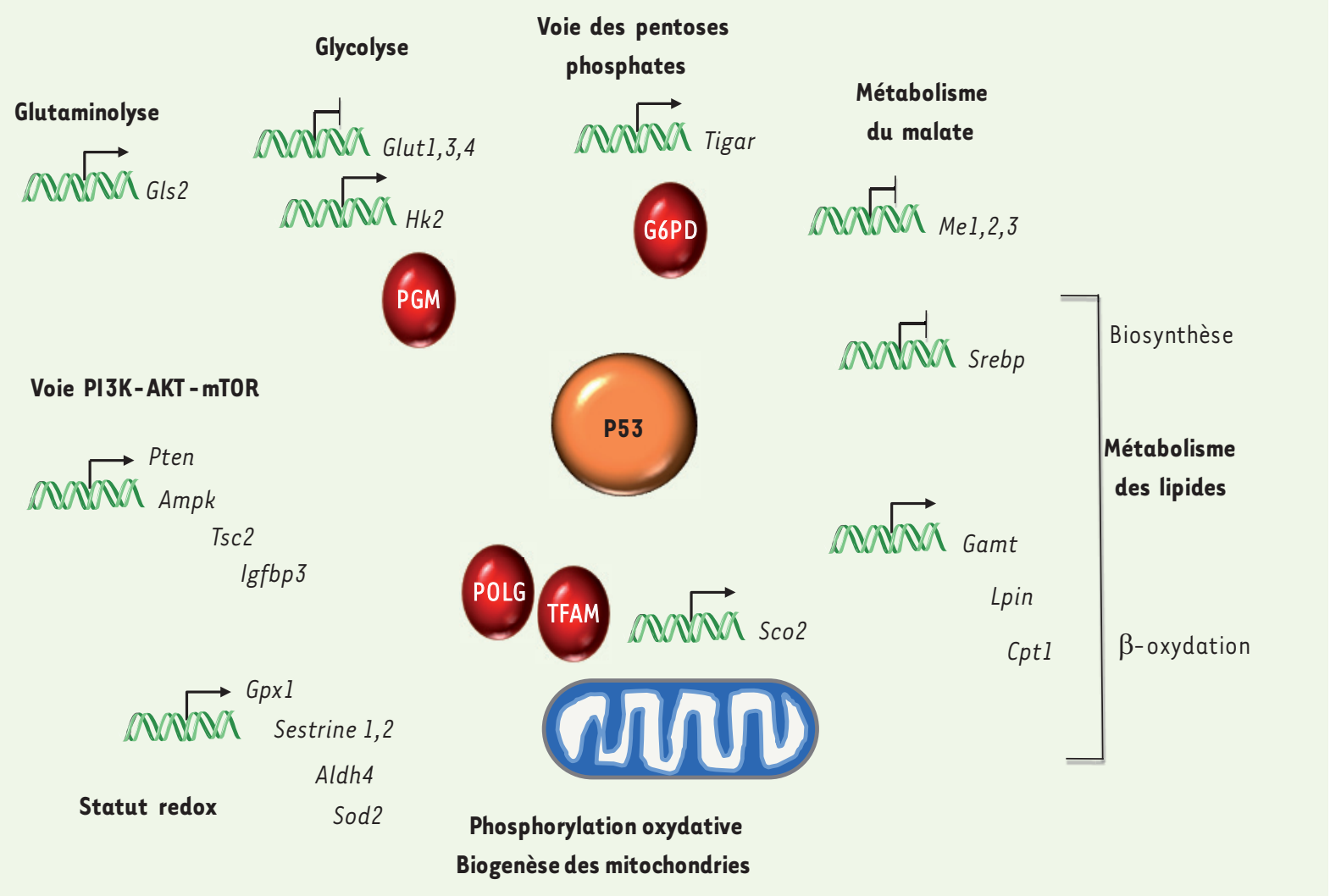

Figure 1. Représentation schématique des différents effets métaboliques régulés par p53. La protéine p53 a des effets sur de nombreuses voies métaboliques, telles que la glycolyse, la branche oxydative de la voie des pentoses phosphates, la glutaminolyse, le métabolisme du malate, la synthèse et la dégradation des acides gras, mais également sur la biosynthèse des mitochondries et l'activité de la chaîne respiratoire, ou encore sur le statut redox. Par ailleurs, p53 régule la transcription de plusieurs éléments clés de la voie PI3K-AKT-mTOR, une voie essentielle du contrôle du métabolisme. Les effets non transcriptionnels (effets sur la stabilité protéique ou interactions protéine-protéine directes) sont représentés en rouge. Gls2 : glutamine synthase 2 ; Glut : transporteur du glucose ; Tigar : tp53-induced glycolysis and apoptosis regulator ; Srebp : sterolregulatory element-binding protein; Gamt : guanidinoacétate $\mathrm{N}$-méthyltransférase ; Ipin l : lipin 1 ; Cptl : carnitine palmitoyl transférase 1 ; Me : enzyme malique ; Pten : phosphatase and tensin homolog; Ampk: AMP-kinase ; Tsc2 : tuberous sclerosis 2 ; Igfbp3 : IGF-binding protein 3 ; Gpxl : glutathion peroxidase 1 ; Aldh4 : aldéhyde déshydrogénase 4 ; Sod2 : superoxyde dismutase 2 ; Sco2 : synthesis of cytochrome c oxidase ; POLG : DNA polymerase subunit gamma; PGM : phosphoglucose mutase ; TFAM : transcription factor A mitochondrial.

source de production d'énergie par la mitochondrie ( $\beta$-oxydation des acides gras) dans des situations de carence en glucose ou au cours d'une période de jeûne, notamment en régulant la transcription de Gamt (guanidinoacetate N-methyltransferase) [14], de Lpin (lipo-protein ice nucleator) [15] et de plusieurs carnitine acyl transferases (Crot, CptlA et CptlC qui conjuguent des groupements carnitine aux acides gras, une étape limitante de leur transport dans la mitochondrie) [16]. p53 a également des effets sur la biosynthèse des lipides en régulant négativement SREBPI (sterol regulatory element-binding protein 1 ), un facteur de transcription agissant sur plusieurs gènes essentiels à la biosynthèse des lipides comme Fasn (fatty acid synthase) ou Acly (ATP-citrate lyase) [17]. Par ailleurs, en réprimant la transcription des gènes codant pour les enzymes maliques 1 et 2 (MEl et -2), p53 est un régulateur important de la production d'équivalents réducteurs, tels que le NADPH qui participe à la biosynthèse des lipides [18].

\section{Autres voies métaboliques}

Certaines études d'immunoprécipation de chromatine réalisées à l'échelle du génome entier suggèrent que p53 (et les autres membres de sa famille) intervient vraisemblablement dans de très nombreuses voies métaboliques. Parmi celles-ci, on trouve la glutaminolyse par l'intermédiaire de la régulation de GLS2 (glutamine synthase 2) $[19,20]$ ou encore la voie de synthèse de la sérine [21].

Si le programme transcriptionnel faisant intervenir p53 dans le métabolisme apparaît de plus en plus complexe, ses effets sur le métabolisme impliquent également des activités non transcriptionnelles, comme l'illustrent les effets sur la stabilité protéique de la phosphoglycérate mutase [22], une enzyme intervenant dans l'une des dernières étapes de la glycolyse, ou encore l'inhibition 


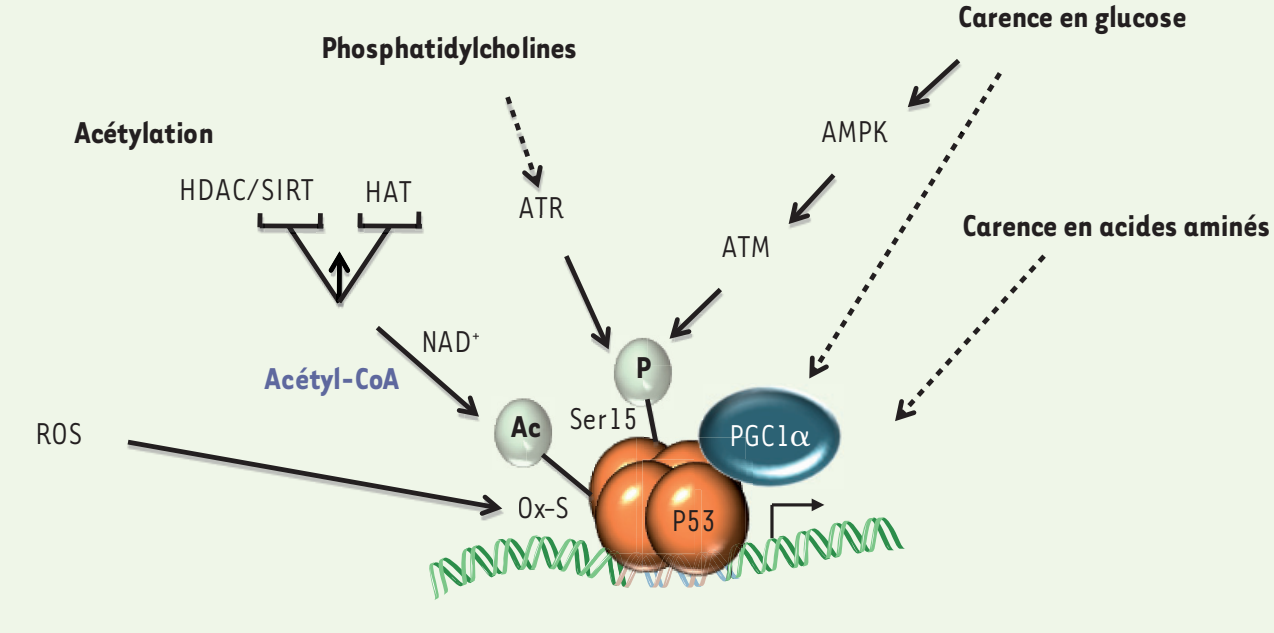

Figure 2. Représentation schématique des différentes perturbations métaboliques régulant les activités de p53. L'activité de la protéine p53 est régulée en réponse à différents types de perturbations métaboliques, telles que la carence en glucose ou en certains acides aminés (notamment la sérine), un changement de concentration de certains phospholipides membranaires (notamment les phosphatidylcholines conjugués à des acides gras insaturés), ou encore les ROS. Ces

perturbations métaboliques peuvent induire des modifications post-traductionnelles de p53, telles que la phosphorylation sur la sérine 15 (sérine 18 chez la souris) ou l'acétylation de la protéine $p 53$, deux modifications réversibles associées à l'activation des fonctions de p53. L'acétylation de 53 est finement régulée par plusieurs acétyltransférases (HAT) ou déacétylases (HDAC ou sirtuines) dont l'activité dépend de cofacteurs (acétyl-CoA, $\left.N A D^{+}\right)$. Ces changements métaboliques peuvent également modifier l'interaction de $p 53$ avec certains corégulateurs transcriptionnels, tels que PGCl $\alpha$. HDAC : histone déacétylase ; SIRT : sirtuin ; HAT : histone acétyltransférase ; ATM : ataxia telangiectasia mutated ; ATR : ATM and Rad3-related; AMPK : AMP-kinase; PGCl $\alpha$ : peroxisome proliferator-activated receptor $\gamma$ coactivator $1 \alpha$.

directe de l'activité enzymatique de la glucose 6-phosphate déshydrogenase (G6PD) [23]. L'activité de la G6PD constituant une des étapes limitantes de la voie des pentoses phosphates, la régulation négative de p53 sur la G6PD devrait, en théorie, s'opposer aux effets de p53 sur TIGAR qui oriente le flux glycolytique vers cette voie métabolique. D'autres travaux seront nécessaires pour mieux comprendre le rationnel biologique de ces effets apparemment paradoxaux et les situations physiopathologiques dans lesquelles ils interviennent.

\section{Contrôle du programme métabolique régulé par p53}

Une question importante encore non résolue concerne les mécanismes moléculaires par lesquels p53 parvient à coordonner des réponses transcriptionnelles permettant à la cellule de s'adapter à son environnement. Par définition, ces réponses adaptatrices sont nombreuses au cours de la vie d'une cellule ou d'un organisme, parfois cycliques, et dans l'immense majorité des cas elles ne doivent pas se traduire par une réponse létale. De fait, il apparaît nécessaire que les activités métaboliques associées à p53 soient coordonnées avec ses autres fonctions, comme celles conduisant à la mort cellulaire. Une des clés de cette coordination fait intervenir le coactivateur transcriptionnel PGCl $\alpha$ (peroxisome proliferator-activated receptor $\gamma$ coactivator $1 \alpha$ ), un régulateur important du métabolisme et de la biosynthèse des mitochondries avec lequel p53 interagit directement pour activer des gènes clés du métabolisme, tels que Tigar, Sco2 ou sestrin 2 [24]. Les liens entre p53 et PGCl $\alpha$ sont complexes, car dans des conditions de stress métabolique prolongé, la dégradation de PGCl $\alpha$ est corrélée à l'induction de gènes cibles de $p 53$ qui induisent la mort cellulaire, tels que Bax (Bcl-2-associated X protein), Puma ( $p 53$ upregulated modulator of apoptosis) ou Noxa (phorbol-12-myristate-13-acetate-induced protein 1). Par ailleurs, un nombre croissant d'études suggèrent que certaines modifications post-traductionnelles de $p 53$ permettent de spécifier des programmes transcriptionnels impliqués dans la régulation du métabolisme. C'est le cas de la phosphorylation de la sérine 15 de p53 (sérine 18 chez la souris) qui est activée en réponse à une carence en glucose par un mécanisme déclenché par la kinase AMPK et qui fait intervenir en aval la kinase ATM (ataxia telangiectasia mutated) [15, 25]. Bien que certains modèles murins génétiquement modifiés confirment l'importance de la phosphorylation de ce résidu dans le contrôle du métabolisme in vivo [25, 26], il ne fait aucun doute que d'autres déterminants encore non identifiés (cofacteurs, autres modifications post-traductionnelles, variants d'épissage, etc.) contribuent à la réponse spécifique de p53 vers un programme impliqué dans le contrôle du métabolisme.

\section{La protéine p53 est régulée par différents stress métaboliques}

L'activité de p53 est régulée par divers changements métaboliques (Figure 2), tels que le changement du statut redox, la carence en nutriments (glucose, acides aminés), ou encore la concentration en $N A D^{+}$. Les liens 
entre les ROS (reactive oxygen species) et p53 sont multiples (pour revue, voir [27]). D'une part, le changement du statut redox active ou bloque p53 selon les situations expérimentales et, d'autre part, p53 possède des cystéines et des tyrosines réactives aux ROS, qui influencent sa liaison à l'ADN. Par ailleurs, l'acétylation de p53 qui est associée à une augmentation de son activité transcriptionnelle est finement contrôlée par une balance entre plusieurs acétyltransférases et dé-acétylases (dont certaines sirtuines) dont l'activité dépend respectivement des concentrations cellulaires en acétyl-CoA et en $N A D^{+}$. De façon intéressante, certaines enzymes du métabolisme (notamment la malate déhydrogénase 1) semblent capables de moduler directement les activités transactivatrices de p53 en réponse à une carence en glucose [28]. À l'inverse, la perturbation d'autres enzymes du métabolisme, telles que les enzymes maliques MEl et -2 , induit l'activation de p53 par deux mécanismes distincts, impliquant respectivement la modulation de l'expression de MDM2 et l'activation de l'AMPK [29]. Enfin, sous le contrôle de la kinase ATR, des changements de concentration en phospholipides membranaires, en particulier les phosphatidylcholines couplés à des acides gras poly-insaturés, induisent la phosphorylation de p53 sur la sérine 15 et son activation [30].

\section{Quelles sont les fonctions in vivo des réponses métaboliques régulées par $\mathrm{p} 53$}

Si les multiples effets de p53 sur le métabolisme ont d'abord été identifiés dans des modèles de lignées cellulaires, de nombreux modèles de souris génétiquement modifiées pour le locus $p 53$ ont depuis permis de confirmer in vivo la relevance physiologique de certaines de ces observations dans l'homéostasie tissulaire, le développement tumoral ou le vieillissement cellulaire. Plusieurs études illustrent les effets de l'inactivation du gène $p 53$ sur le métabolisme glucidique et lipidique et ses conséquences sur la fonction de différents tissus adultes, par des effets à la fois directs et indirects. Ainsi, les souris adultes invalidées pour p53 (p53-KO) développent un défaut de néoglucogenèse hépatique à jeun [24]. L'inactivation de $p 53$ semble également affecter l'endurance et la force musculaire, un phénotype qui est corrélé à une diminution significative du nombre des mitochondries et de leur activité respiratoire dans le muscle squelettique [31]. Certaines données récentes suggèrent un rôle particulier de p53 sur le tissu adipeux, un effet qui pourrait illustrer un rôle important de p53 dans la protection des adipocytes vis-à-vis des effets toxiques de l'accumulation d'acides gras (un phénomène appelé lipotoxicité). De fait, les animaux $p 53 \mathrm{KO}$ prennent plus de poids lorsqu'ils sont soumis à un régime hypercalorique [32]. Plusieurs équipes ont confirmé que des fibroblastes primaires ou des progéniteurs d'adipocytes isolés à partir des souris $p 53 \mathrm{KO}$ accumulent plus de lipides au cours de la différenciation adipocytaire ex vivo $[18,33]$. Cependant, différents modèles génétiquement modifiés illustrent la complexité des effets de p53 sur le tissu adipeux. Ainsi, si l'inactivation totale ou ciblée de $p 53$ dans le tissu adipeux tend à normaliser la tolérance au glucose et la sensibilité à l'insuline que développent les animaux sous régime hypercalorique
$[26,34]$, d'autres modèles indiquent que les effets de p53 vis-à-vis de ces phénotypes métaboliques sont plus complexes et dépendent vraisemblablement de la nature des isoformes de p53 mises en jeu, de leur niveau d'expression et de certains effets paracrines associés aux cellules inflammatoires infiltrantes [25, 26, 34].

Les effets métaboliques de $\mathrm{p} 53$ pourraient avoir des rôles importants dans la survenue de certaines pathologies associées au vieillissement. Ainsi, via le contrôle de l'expression de certaines lipoprotéines synthétisées par le foie, il est possible que $p 53$ constitue une ligne de défense contre l'athérosclérose [16]. Cependant, certains des effets provieillissement de $\mathrm{p} 53$ ont récemment été couplés à des effets métaboliques faisant intervenir le corégulateur transcriptionnel PGCl $\alpha$ [35], soulignant une fois de plus la complexité des effets métaboliques associés à $p 53$.

Enfin, une des questions essentielles concerne l'importance des réponses métaboliques régulées par p53 au cours de la transformation cellulaire. Certains travaux montrent que la perturbation du statut redox des cellules dans lesquelles p53 est inactivée participe à l'instabilité génétique et joue un rôle important au cours de la leucémogenèse [36]. De façon beaucoup plus surprenante, des travaux récents ont suggéré que les fonctions suppresseur de tumeurs de $p 53$ sont principalement associées à sa capacité à réguler le métabolisme, plutôt que la sénescence, l'arrêt du cycle ou l'apoptose [37].

\section{Conclusions}

Les données de nombreux laboratoires renforcent l'idée selon laquelle les rôles de p53 dans le métabolisme cellulaire sont multiples. Plus de 30 ans après sa découverte, une nouvelle ère concernant l'étude de ce suppresseur de tumeurs aux multiples facettes vient de s'ouvrir ; elle permettra sûrement de mieux comprendre, dans les années à venir, certains de ses effets sur I'homéostasie tissulaire, le vieillissement cellulaire [38, 39] et le développement tumoral [40]. $\diamond$

\section{SUMMARY}

Role of the 53 tumor suppressor in metabolism The p53 tumor suppressor is an essential downstream effector of various cellular stress response pathways that is functionally inactivated in most, if not all, tumors. Since its discovery more than 30 years ago, its role in the control of cell proliferation, senescence and cell survival has been widely described. However, growing evidences from several laboratories indicate that p53 has important transcriptional and non-transcriptional 
functions in the control of metabolism, including the regulation of glycolysis, glutaminolysis or mitochondrial respiration. Originally identified using in vitro cellular models, this previously underestimated role of $p 53$ has been confirmed in vivo in various genetically engineered mouse models. These recent data suggest that $p 53$ functions in various metabolic pathways significantly contribute to its role in adult tissue homeostasis, aging as well as tumor suppression. $\diamond$

\section{LIENS D'INTÉRÊT}

Les auteurs déclarent n'avoir aucun lien d'intérêt concernant les données publiées dans cet article.

\section{RÉFÉRENCES}

1. Feng Z, Hu W, de Stanchina $\varepsilon$, et al. The regulation of AMPK1, TSC2, and PTEN expression by p53: stress, cell and tissue specificity, and the role of these gene products in modulating the IGF-1AKT-mTOR pathways. Cancer Res $2007 ; 67: 3043-53$.

2. Stambolic V, MacPherson D, Sas D, et al. Regulation of PTEN transcription by p53. Mol Cell 2001; $8: 317-25$.

3. Velasco-Miguel S, Buckbinder L, Jean P, et al. PA26, a novel target of the $p 53$ tumor suppressor and member of the GADD family of DNA damage and growth arrest inducible genes. Oncogene $1999 ; 18: 127-37$

4. Budanov AV, Shoshani T, Faerman A, et al. Identification of a novel stress-responsive gene Hi95 involved in regulation of cell viability. Oncogene $2002 ; 21: 6017-31$.

5. Budanov AV, Karin M. p53 Target genes sestrinl and sestrin2 connect genotoxic stress and mTOR signaling. Cell $2008 ; 134: 451-60$.

6. Gottlieb TM, Leal JFM, Seger R, et al. Cross-talk between Akt, $\mathrm{p} 53$ and Mdm2: possible implications for the regulation of apoptosis. Oncogene $2002 ; 21: 1299-303$.

7. Schwartzenberg-Bar-Yoseph F. The tumor suppressor $\mathrm{p} 53$ down-regulates glucose transporters GLUTl and GLUT4 gene expression. Cancer Res $2004 ; 64$ : 2627-33.

8. Kawauchi K, Araki K, Tobiume K, et al. p53 regulates glucose metabolism through an IKK-NF-KB pathway and inhibits cell transformation. Nat Cell Biol $2008 ; 10: 611-8$.

9. Bensaad K, Tsuruta A, Selak MA, et al. TIGAR, a p53-inducible regulator of glycolysis and apoptosis. Cell 2006; $126: 107-20$.

10. Matoba S. p53 regulates mitochondrial respiration. Science 2006 ; 312 : 1650-3.

11. Yoshida Y, Izumi H, Torigoe T, et al. P53 physically interacts with mitochondrial transcription factor A and differentially regulates binding to damaged DNA. Cancer Res $2003 ; 63: 3729-34$.

12. Achanta $G$, Sasaki R, Feng L, et al. Novel role of $p 53$ in maintaining mitochondrial genetic stability through interaction with DNA Pol gamma. EMBOJ 2005 ; 24 : 3482-92.

13. Mathupala SP, Heese C, Pedersen PL. Glucose catabolism in cancer cells. The type II hexokinase promoter contains functionally active response elements for the tumor suppressor p53. J Biol Chem 1997 ; 272 : 22776-80.

14. Ide T, Brown-Endres L, Chu K, et al. GAMT, a p53-inducible modulator of apoptosis, is critical for the adaptive response to nutrient stress. Mol Cell $2009 ; 36: 379-92$.

15. Assaily W, Rubinger DA, Wheaton K, et al. ROS-mediated $\mathrm{p} 53$ induction of Lpinl regulates fatty acid oxidation in response to nutritional stress. Mol Cell $2011 ; 44: 491-501$.

16. Goldstein I, Ezra 0, Rivlin N, et al. p53, a novel regulator of lipid metabolism pathways. J Hepatol $2012 ; 56: 656-62$.

17. Yahagi N, Shimano H, Matsuzaka T, et al. 553 activation in adipocytes of obese mice.J Biol Chem $2003 ; 278: 25395-400$.

18. Jiang P, Du W, Mancuso A, et al. Reciprocal regulation of $\mathrm{p} 53$ and malic enzymes modulates metabolism and senescence. Nature $2013 ; 493: 689-93$.

19. Hu W, Zhang C, Wu R, et al. Glutaminase 2, a novel $p 53$ target gene regulating energy metabolism and antioxidant function. Proc Natl Acad Sci USA $2010 ; 107: 7455-60$.
20. Suzuki S, Tanaka T, Poyurovsky MV, et al. Phosphate-activated glutaminase (GLS2), a p53-inducible regulator of glutamine metabolism and reactive oxygen species. Proc Natl Acad Sci USA 2010 ; 107 : 7461-6.

21. Maddocks ODK, Berkers CR, Mason SM, et al. Serine starvation induces stress and p53-dependent metabolic remodelling in cancer cells. Nature 2013; $493: 542-6$.

22. Kondoh $\mathrm{H}$, Lleonart ME, Gil J, et al. Glycolytic enzymes can modulate cellular life span. Cancer Res $2005 ; 65: 177-85$.

23. Jiang P, Du W, Wang X, et al. p53 regulates biosynthesis through direct inactivation of glucose-6-phosphate dehydrogenase. Nat Cell Biol 2011 ; $13: 310-6$.

24. Sen N, Satija YK, Das S. PGC-lalpha, a key modulator of $p 53$, promotes cell survival upon metabolic stress. Mol Cell 2011; $44: 621-34$.

25. Armata HL, Golebiowski D, Jung DY, et al. Requirement of the ATM/p53 tumor suppressor pathway for glucose homeostasis. Mol Cell Biol 2010 ; 30 : 5787 94.

26. Franck D, Tracy L, Armata HL, et al. Glucose tolerance in mice is linked to the dose of the $p 53$ transactivation domain. Endocr Res $2012 ; 38: 139-50$.

27. Maillet $A$, Pervaiz S. Redox regulation of $p 53$, redox effectors regulated by p53: a subtle balance. Antioxid Redox Signal $2012 ; 16$ : 1285-94.

28. Lee SM, Kim JH, Cho EJ, et al. A nucleocytoplasmic malate dehydrogenase regulates $p 53$ transcriptional activity in response to metabolic stress. Cell Death Differ $2009 ; 16: 738-48$.

29. Jiang D, Attardi LD. Engaging the $p 53$ metabolic brake drives senescence. Cell Res $2013 ; 23: 739-40$.

30. Zhang XH, Zhao C, Ma ZA. The increase of cell-membranous phosphatidylcholines containing polyunsaturated fatty acid residues induces phosphorylation of $p 53$ through activation of ATR. J Cell Sci 2007 ; $120: 4134-43$.

31. Saleem A, Adhihetty PJ, Hood DA. Role of $p 53$ in mitochondrial biogenesis and apoptosis in skeletal muscle. Physiol Genomics 2009 ; 37 : 58-66.

32. Molchadsky A, Ezra 0, Amendola PG, et al. p53 is required for brown adipogenic differentiation and has a protective role against diet-induced obesity. Cell Death Differ 2013; $20: 744-83$.

33. Molchadsky A, Shats I, Goldfinger N, et al. p53 plays a role in mesenchymal differentiation programs, in a cell fate dependent manner. Preiss T, Ed. PloS One 2008 ; 3 : e3707.

34. Minamino T, Orimo M, Shimizu I, et al. A crucial role for adipose tissue $p 53$ in the regulation of insulin resistance. Nat Med $2009 ; 15$ : 1082-7.

35. Sahin $\varepsilon$, Colla S, Liesa M, et al. Telomere dysfunction induces metabolic and mitochondrial compromise. Nature $2011 ; 470: 359-65$.

36. Sablina AA, Budanov AV, llyinskaya GV, et al. The antioxidant function of the p53 tumor suppressor. Nat Med $2005 ; 11$ : 1306-13.

37. Li T, Kon N, Le Jiang, et al. Tumor suppression in the absence of p53mediated cell-cycle arrest, apoptosis, and senescence. Cell $2012 ; 149$ : 1269-83.

38. Brondello JM, Prieur A, Philipot D, et al. La sénescence cellulaire. Med Sci (Paris) $2012 ; 28: 288-96$.

39. Bischof 0 , Dejean $A$, Pineau P. Une re-vue de la sénescence cellulaire. Med Sci (Paris) $2009 ; 25: 153-60$.

40. Floquet $C$, Rousset JP, Bidou L. La réactivation par translecture du gène p53 possédant une mutation non-sens induit l'apoptose de cellules cancéreuses. Med Sci (Paris) $2011 ; 27:$ 585-6.

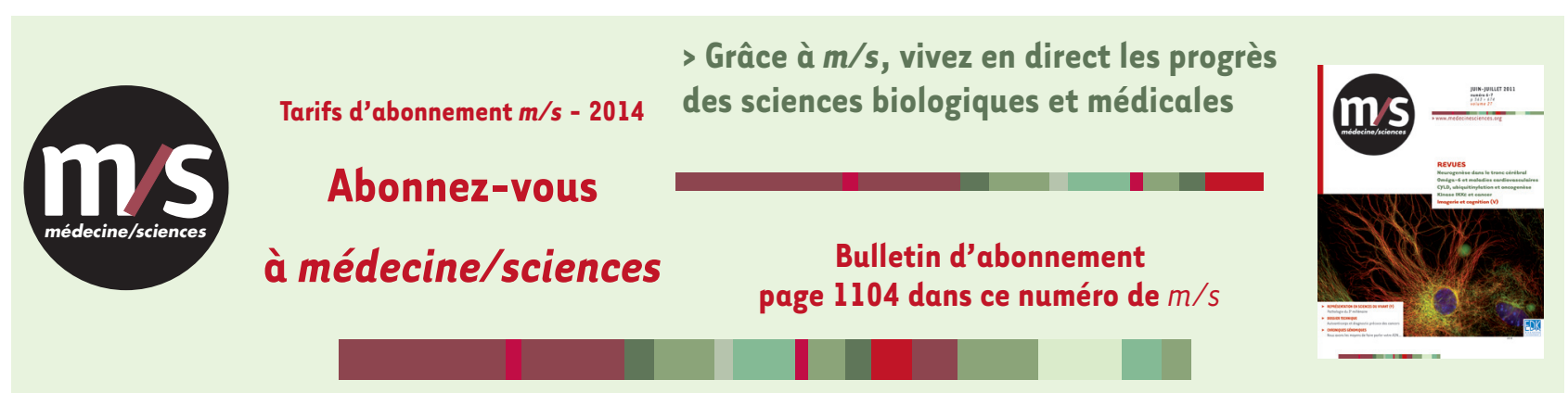

TIRÉS À PART

L. Le Cam 